\title{
Aerobic microbial manufacture of nanoscale selenium: exploiting nature's bio-nanomineralization potential
}

\author{
N. Tejo Prakash $\cdot$ Neetu Sharma $\cdot$ Ranjana Prakash • \\ Kuldeep K. Raina $\cdot$ Jonathan Fellowes $\cdot$ Carolyn I. Pearce • \\ Jonathan R. Lloyd · Richard A. D. Pattrick
}

Received: 23 June 2009/Revised: 17 July 2009/Accepted: 20 July 2009/Published online: 19 August 2009

(C) Springer Science+Business Media B.V. 2009

\begin{abstract}
The potential of the environment to yield organisms that can produce functional bionanominerals is demonstrated by selenium-tolerant, aerobic bacteria isolated from a seleniferous rhizosphere soil. An isolate, NS3, was identified as a Bacillus species (EU573774.1) based on morphological and 16S rRNA characterization. This strain reduced Se(IV) under aerobic conditions to produce amorphous $\alpha \operatorname{Se}(0)$ nanospheres. A room-temperature washing treatment was then employed to remove the biomass and resulted in the production of clusters of hexagonal $\mathrm{Se}(0)$ nanorods. The $\mathrm{Se}(0)$ nanominerals were analyzed using electron microscopy and X-ray diffraction techniques. This Bacillus isolate has the potential to be used both in the neutralizing of toxic Se(IV) anions in the
\end{abstract}

N. Tejo Prakash $(\bowtie) \cdot$ N. Sharma

Department of Biotechnology and Environmental

Sciences, Thapar University, Patiala, India

e-mail: ntejoprakash@thapar.edu

R. Prakash

School of Chemistry and Biochemistry,

Thapar University, Patiala, India

K. K. Raina

School of Physics and Material Sciences,

Thapar University, Patiala, India

J. Fellowes - C. I. Pearce - J. R. Lloyd - R. A. D. Pattrick

School of Earth, Atmosphere and Environmental

Sciences, University of Manchester, Manchester, UK environment and in the environmentally friendly manufacture of nanomaterials.

Keywords Bacillus · Nanospheres ·

Reduction · Rosettes - Selenium

\section{Introduction}

A variety of microorganisms, bacteria, yeast, fungi and algae, can adsorb and accumulate metals but only a few groups can selectively reduce metal ions to produce nano-scale mineral phases (Oremland et al. 2004). These organisms have the unique ability to produce inorganic phases of constant chemical composition and size (Cui and Gao 2003; Pearce et al. 2008). The majority of studies on the biogenesis of nano-Se particles have concentrated on anaerobic systems that have certain limitations, such as culture conditions and isolate characteristics that make optimization and scale-up in bio-manufacturing processes challenging. Selenium-tolerant aerobic organisms, however, provide the opportunity to overcome these limitations in the biosynthetic process. The results presented here show the Se reducing ability of a bacterial isolate, Bacillus sp. NS3 (EU573774.1) obtained from a seleniferous rhizospheric soil in the North-Western State of Punjab, India. The isolate tolerates selenium oxyanions and generates selenium 
nanoparticles, thus combining the detoxification of oxidized seleniferous environments with the biotechnological production of nanomaterials.

It is the great potential of nano-scale $\operatorname{Se}(0)$ phases in electronic, optical, catalytic and medical application that has led to extensive investigations of the production and post-preparative modification of these materials by various inorganic routes (Pearce et al. 2008; Takahashi et al. 2006; Zhou and Zhu 2006). These include, solid-solution-solid transformation from amorphous $\mathrm{Se}(a-\mathrm{Se})$ colloids to $t$-Se nanowires (Gates et al. 2000, 2002a) and sonochemistry based synthesis and transformation of $a$-Se to $t$-Se nanowires (Gates et al. 2002b). In this study, we report on a solvent-based post-preparative treatment process to convert biomass-associated amorphous Se nanospheres, produced by the aerobic environmental isolate, Bacillus sp. (NS3; EU573774.1), into 'clean' crystalline Se nanorods for potential photovoltaic applications.

\section{Materials and methods}

Isolation and characterization

The isolate under study was obtained from soil collected from seleniferous belt bordering the Nawanshahr-Hoshiarpur region in North-west India $\left(75^{\circ} 55 \mathrm{E} ; 31^{\circ} 56 \mathrm{~N}\right)$ through standard enrichment procedures (Focht 1994). This strain was selected based on its potential to tolerate selenium as selenite $\left(\mathrm{SeO}_{3}{ }^{2-}\right)$. Growth experiments were conducted with the test strain to examine the Se tolerance of both aerobic and anaerobic cultures in tryptone soy broth (TSB), supplemented with $\mathrm{Na}_{2} \mathrm{SeO}_{3}(5 \mathrm{mM})$. Morphological analysis and gram staining were carried out by applying standard protocols (Krieg et al. 1984). Phylogenetic affiliation of this isolate was done by sequencing of the $16 \mathrm{~S}$ rRNA gene. PCR amplification of the partial 16S rRNA gene was carried out using universal primers (8f and 1492r). The PCR conditions were as follows: Preheating at $94^{\circ} \mathrm{C}$ for $300 \mathrm{~s}, 35$ cycles of $94^{\circ} \mathrm{C}$ for $60 \mathrm{~s}, 55^{\circ} \mathrm{C}$ for $30 \mathrm{~s}$ and $72^{\circ} \mathrm{C}$ for $30 \mathrm{~s}$ and a final extension at $71^{\circ} \mathrm{C}$ of $300 \mathrm{~s}$. The PCR product was eluted and purified using a gel elution kit (Sigma), ligated to a linearized vector pTZ57 R/T (Fermentas) and transformed in competent Escherichia coli DH5- $\alpha$ cells.
The cloned gene was then amplified and sequenced at the DNA sequencing facility, University of Delhi, India. The computational tool ClustalW (Thomson et al. 1994) was used to align and compare the $16 \mathrm{~S}$ rRNA gene sequences of closely related to species of Bacilli. Phylogenetic analyses were conducted using the MEGA 4.0.1 software package (Tamura et al. 2007).

Growth profile and Se(IV) reduction under aerobic and anaerobic conditions

Erlenmeyer flasks and serum bottles, containing sterile TSB supplemented with $1 \mathrm{mM} \mathrm{Se}$, were inoculated with a culture grown to log phase (based on optical density) so as to examine the growth profile in aerobic and anaerobic conditions. Additional supplementation of $7.5 \mathrm{~g}$ sodium lactate $\mathrm{l}^{-1}$ was provided as an electron donor to the anaerobic cultures. $\mathrm{H}_{2}$ was also passed through the medium in the serum bottles for $5 \mathrm{~m}$ in as an additional electron donor. Positive (TSB with inoculum and without selenium) and negative (TSB with selenium and without inoculum) controls were maintained both for aerobic and anaerobic conditions. The negative control was used to assess the potential for chemical reduction of Se by the medium. Growth of the cultures was observed by measuring the $\mathrm{OD}_{600}$ over $24 \mathrm{~h}$. The removal of selenite was determined by measuring the concentration of the oxyanion in the cell free supernatant (CFS) using ion chromatography (IC - Dionex DX 600) with $9 \mathrm{mM} \mathrm{Na}_{2} \mathrm{CO}_{3}$ as the mobile phase and an AS 9-HC column at $16.92 \mathrm{MPa}$ back-pressure. The samples were diluted appropriately and introduced through a GP50 gradient pump to a CD 20 conductivity detector.

\section{Characterization of reduced selenium}

The solid fraction of the inocula (reduced selenium phases and biomass) were separated by centrifugation at $4000 \times g$ for $15 \mathrm{~min}$. These samples were then imaged using an environmental scanning electron microscope (ESEM, Phillips XL) employing a GSE detector at 0.4-0.7 Torr. Energy dispersive X-ray (EDX) spectroscopy was performed at $16-20 \mathrm{kV}$ using spot size of $200 \mathrm{~nm}$ and a counting for $100 \mathrm{~s}$ to provide qualitative chemical characterization of the phases produced. The biomass with the Se-phases was then subjected to sequential washing and 
centrifugation $(4000 \times g$ for $5 \mathrm{~min})$ steps in $70,80,90$ and $99 \%$ ethanol/water $(90: 1 \mathrm{v} / \mathrm{v})$, followed by a final washing step in chloroform/methanol $(1: 1 \mathrm{v} / \mathrm{v})$. The products were also examined using ESEM/EDX and further examined using transmission electron microscopy (TEM, Philips, CM-200). X-ray diffraction analysis using a Bruker D8 Advance X-ray diffractometer (Bruker AXS Ltd., Coventry, UK) with a $\mathrm{Cu}$ $K \alpha 1$ source was used to determine the crystal structure of the selenium phases produced.

\section{Results}

Characterization of the isolate

Morphological and phylogenetic analyses suggested that the isolate, NS3, was a Bacillus strain (EU573774.1); the isolate stained Gram-positive and was rod-shaped. In addition the 16S rRNA gene of the isolate shares $99 \%$ similarity (on 1,513 bp) with the 16S rRNA gene sequences from Bacillus thuringiensis, B. anthracis, and B. cereus (Fig. 1). Growth of the isolate under aerobic and anaerobic conditions confirmed the isolate to be a facultative anaerobe.

Selenite reduction under aerobic and anaerobic conditions

The potential of NS3 to transform selenite was assessed by challenging both aerobic and anaerobic cultures with sodium selenite $(1 \mathrm{mM})$. IC data showed a decrease in $\mathrm{Se}(\mathrm{IV})$ concentration in the cell free supernatant, corresponding with growth of the culture under aerobic conditions, as shown by an increase in $\mathrm{OD}_{600 \mathrm{~nm}}$ (Fig. 2). During growth and Se(IV) reduction, production of a red precipitate was observed in the aerobic cultures. Growth and $\mathrm{Se}(\mathrm{IV})$ reduction was limited under anaerobic conditions (Fig. 2). A marginal decrease in $\mathrm{Se}(\mathrm{IV})$ concentration $(\sim 10 \%)$ as a result of chemical reduction by the TSB media was observed in the no-cell controls under anaerobic conditions but not under aerobic conditions (data not shown).

Characterization of selenium nanostructures

ESEM examination of the red cell pellet from the Se-supplemented medium inoculated with the NS3 revealed 100-200 $\mathrm{nm}$ nanospheres associated with the biomass (Fig. 3a). EDX analysis revealed the nanospheres to be made exclusively of Se (Fig. 3b) indicating the formation of $\mathrm{Se}(0)$.

The post-preparative treatment process, involving water/ethanol and chloroform/methanol solvent systems, was employed to isolate the Se nanospheres from the biomass. However, this treatment resulted in the gradual change in the precipitate colour from red to black. ESEM images show that this color change was concurrent with a morphological change from the relatively small $(10-200 \mathrm{~nm})$ nanospheres (Fig. 3a) via hexagonal, faceted and increasingly platy nano-structures (Fig. 4a) to larger (up to
Fig. 1 16S rRNA gene based phylogenetic placement of the isolate as a Bacillus species. Scale bars represent substitutions per site

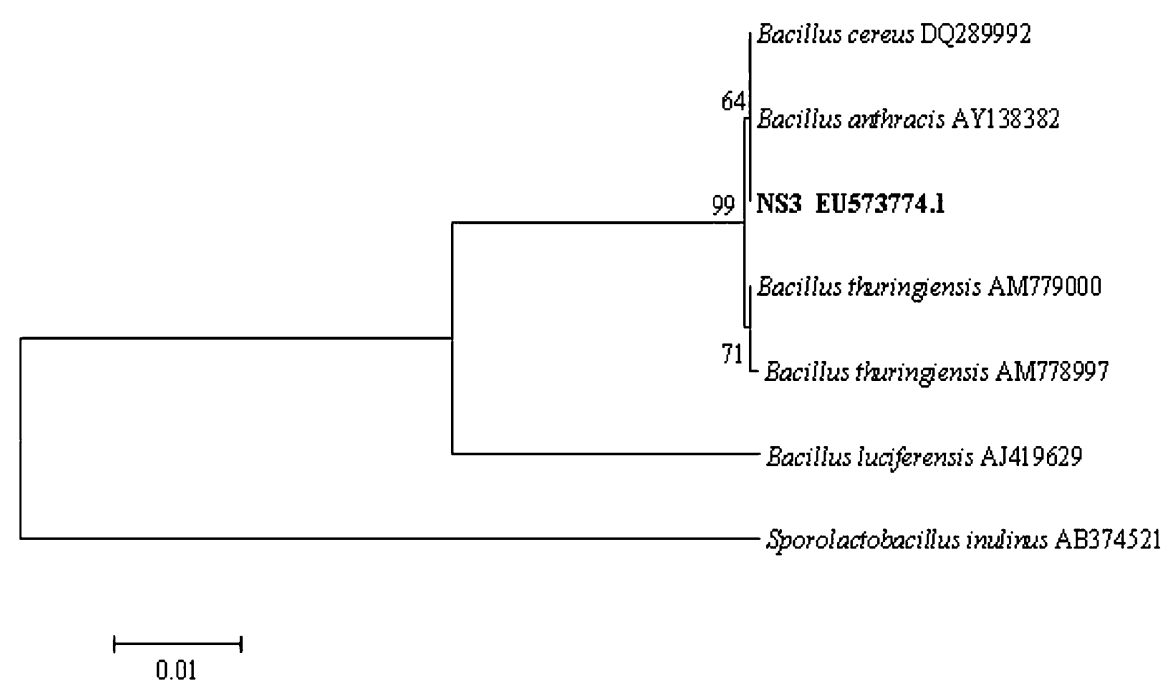




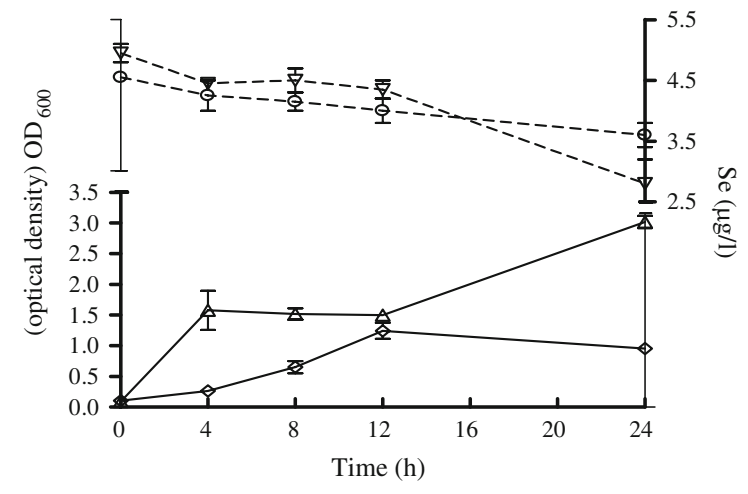

Fig. 2 Change in selenite concentration under aerobic (inverted triangle) or anaerobic (open circle) conditions in cell free supernatant and growth profile under aerobic (open triangle) or anaerobic conditions (open diamond) during $24 \mathrm{~h}$ exposure of Bacillus sp. to $1 \mathrm{mM}$ selenite

$10 \mu \mathrm{m})$ nano-rosettes comprising several crystalline nano-rods (Fig. 5a). EDX analysis indicated that the intermediate nano-structures and the nano-rosettes were also composed solely of selenium (Figs. 4b, 5b).

TEM examination revealed each nano-rod in the rosette to be $\sim 5$ to $10 \mu \mathrm{m}$ length and $0.5-1 \mu \mathrm{m}$ wide (Fig. 6a, b). XRD analysis revealed that the red nano-spheres were amorphous $\operatorname{Se}(0)$ (Fig. 7a). The diffraction pattern of the black Se nano-rosettes was the same as crystalline synthetic hexagonal Se (powder diffraction file 060362, International Centre for Diffraction Data) with characteristic peaks $(2 \theta)$ at 23.42, 29.64 and 43.58 (Fig. 7b). The abnormal intensity of the (100) peak (as compared to that of synthetic $\mathrm{Se}$ ) indicates that the Se nano-rosettes are preferentially orientated along the c axis, the [001] direction.

\section{Discussion}

Amorphous Se nanospheres, such as those produced by NS3, comprise both disordered $\left[-\mathrm{Se}-{ }_{n}\right]$ chains and $\mathrm{Se}_{8}$-rings and are relatively unstable at ambient temperatures (Peled and Hadziioannou 1991; Kasap and Yannacopoulos 1989). However, the biogenic Se nanospheres produced in this study remained stable until the organic component was removed in the postpreparative washing step, suggesting that the biomass was acting as a stabilizing agent. The amorphous red Se nanospheres were then transformed to crystalline
Fig. 3 ESEM image $(-2 \mu \mathrm{m})(\mathbf{a})$ and $\operatorname{EDX}(\mathbf{b})$ of selenium nanospheres associated with the Bacillus sp. biomass
Fig. 4 ESEM image $(-1 \mu \mathrm{m})(\mathbf{a})$ and $\operatorname{EDX}(\mathbf{b})$ of intermediate selenium nano-structures showing hexagonal facet development. The inset shows the platy nanostructures on $1 \mu \mathrm{m}$ scale
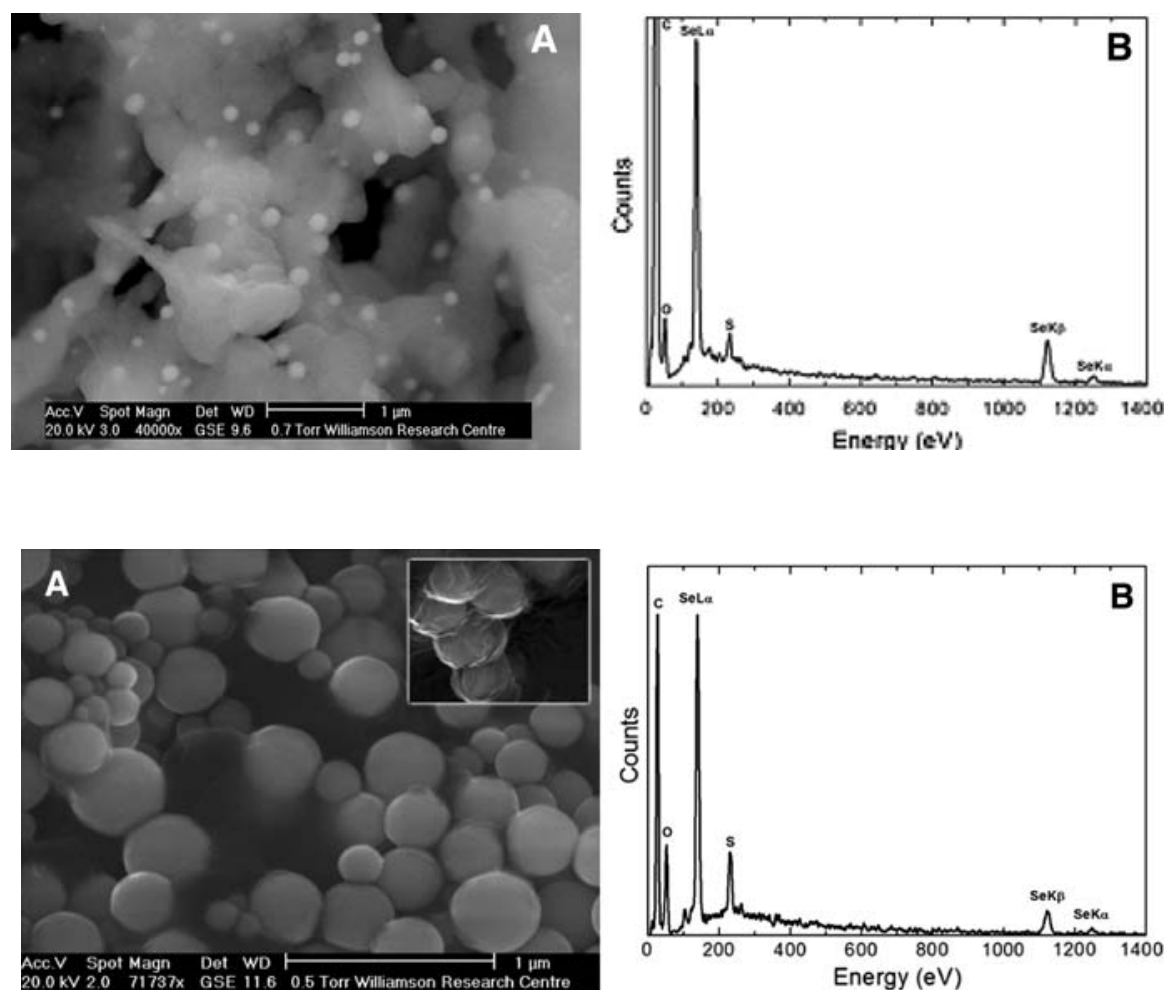
Fig. 5 ESEM image $(-20 \mu \mathrm{m})(\mathbf{a})$ and $\operatorname{EDX}(\mathbf{b})$ of nanowire/rods formed on biomass in the form of rosettes. Inset shows a closer view of a rosette on $5 \mu \mathrm{m}$ scale
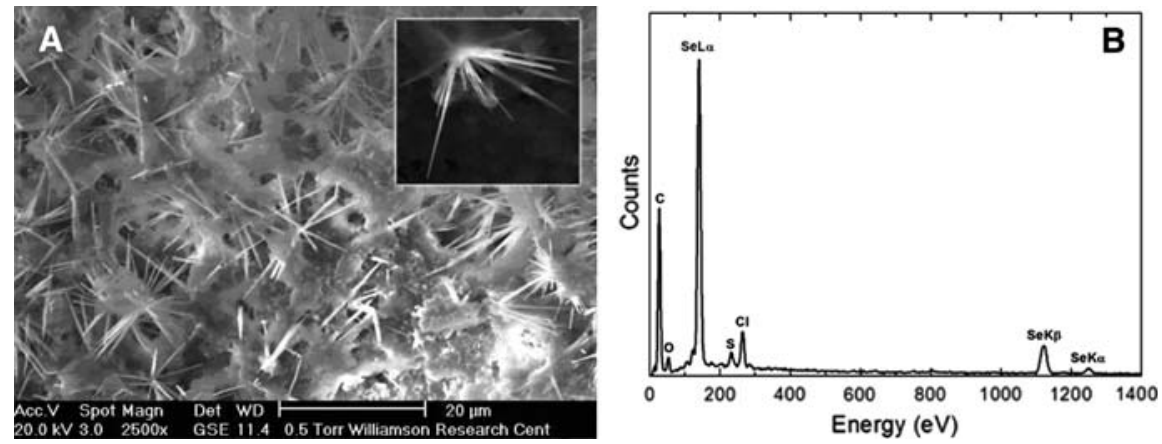

Fig. 6 TEM images of Se nanorods formed as rosettes from the biomassassociated Se nanospheres, showing a some precursor nanospheres $(-1 \mu \mathrm{m})$, and b a detail of a nanorod (scale $50 \mathrm{~nm}$ )
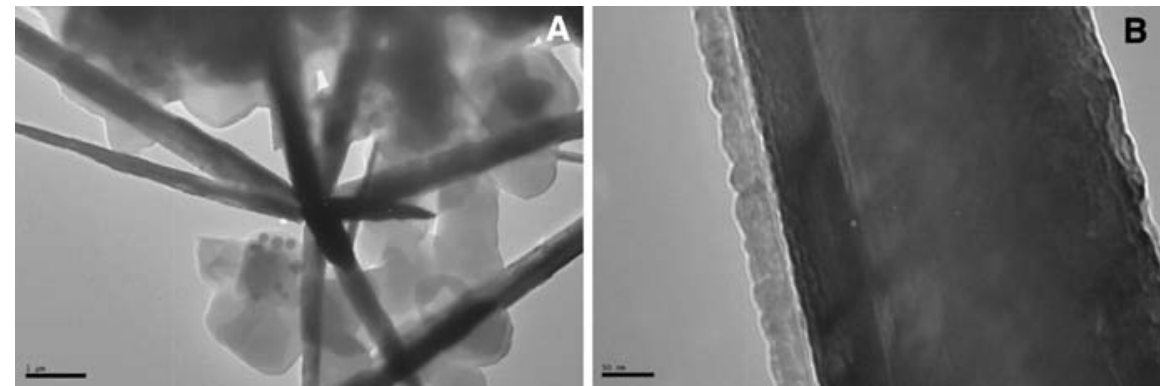

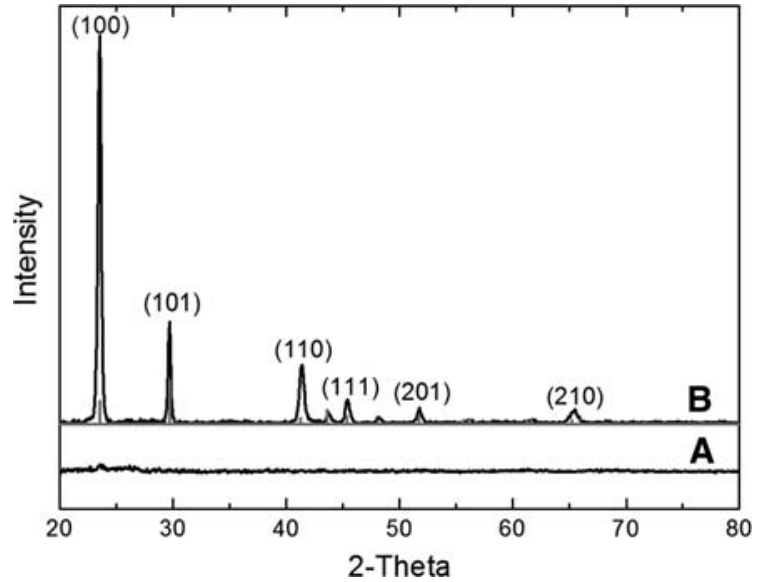

Fig. 7 XRD of amorphous nano-spheres (a) and crystalline nano-rosettes (b). The peak positions and relative intensities of crystalline $t$-Se are shown in grey. The $h k l$ indices of $t$-Se are given in parentheses

hexagonal selenium ( $t$-Se) (Fig. 5a) in the presence of organic solvents, via hexagonal, platy nano-structures (Fig. 4a). The development of Se nanorods in the presence of simple organic solvents has been observed before (Takahashi et al. 2006; Zhou and Zhu 2006; Gates et al. 2002b), although the size of the crystal clusters and the time taken for transformation of red nanospheres to black crystalline nanorods (within
$60 \mathrm{~min}$ ) are exceptional in this case. In previous investigations, the nucleation of the $t$-Se nanorods appeared to occur on hexagonal platelets, which here may have be either neo-formed $t$-Se or an intermediate monoclinic phase (Miyata et al. 1978; Mayers et al. 2001; Gates et al. 2002b; Peled and Hadziioannou 1991). Figures 3a, 4a, 5a in this study show a similar transformation. $t$-Se is very stable and comprises ordered helical $\left[-\mathrm{Se}_{-}\right]$chains linked together by inter-chain Van der Waals forces (Corb et al. 1982; Ren et al. 2004). The nature of the chain structure parallel to the $001 \mathrm{c}$-axis direction in $t$-Se results in one dimensional growth of Se and favors the acicular crystal development seen here. Preferential growth is expected from crystals orientated along the c-axis and the formation of the relatively large radiating rosettes suggests restricted development of $t$-Se nuclei (Zhang et al. 2003; Zhou and Zhu 2006).

\section{Conclusion}

Exploring the capabilities of naturally adapted microbial populations present in contaminated habitats offers the potential to isolate bacterial strains that can assimilate metals, transforming them into stable nanoscale mineral phases. The results of this study 
reveal the potential of a facultative anaerobic, Setolerant environmental isolate (NS3) from seleniferous soil to reduce and transform selenium oxy-anions to elemental selenium. Morphological characterization of the isolate and partial sequencing of its $16 \mathrm{~S}$ rRNA gene indicated that it belongs to the genus Bacillus. Using NS3, it has been demonstrated the biological production of amorphous Se under aerobic conditions offers advantages over chemical processes, in which amorphous Se is produced under environmentally damaging conditions. The effect of the biomass also provides a method of stabilizing this reactive material whereas the washing process provides a facile method for nanorod production. Further investigation of natural environments with anomalous geochemical signatures will provide a range of biomineralizing microbes that can adapt to these chemically hostile environments and have the potential for use in the environmentally friendly manufacture of bionanominerals.

Acknowledgements The authors acknowledge the Chris Boothman, Alastair Bewsher and Steve Caldwell of Williamson Centre for Molecular Environmental Sciences, for facilitating Molecular, ion chromatography and ESEM analysis. NTP acknowledges Erasmus-Mundus Programme for funding MESPOM Visiting Scholarship for carrying out research work at University of Manchester, United Kingdom and Defence Research and Development Organization, India for research grant and fellowship to Ms. Sharma.

\section{References}

Corb BW, Wei WD, Averbach BL (1982) Atomic models of amorphous selenium. J Non-Cryst Solids 53:29-42

Cui D, Gao H (2003) Advance and prospects of bionanomaterials. Biotechnol Prog 19:683-692

Focht DD (1994) Microbiological procedures for biodegradation research. In: Weaver RW, Angle JS, Bottomley PS (eds) Methods of soil analysis, part 2. Microbiological and biochemical properties. Soil Science Society of America, Madison, WI, pp 407-426

Gates B, Yin Y, Xia Y (2000) A solution phase approach to synthesis of uniform nanowires of crystalline selenium with lateral dimensions in the range of $10-30 \mathrm{~nm}$. J Am Chem Soc 122:12582-12583

Gates B, Mayers B, Cattle B, Xia Y (2002a) Synthesis and characterization of uniform nanowires of trigonal selenium. Adv Funct Mater 12:219-227
Gates B, Mayers B, Grossman A, Xia Y (2002b) A sonochemical approach to the synthesis of crystalline selenium nanowires in solutions and on solid supports. Adv Mater 14:1749-1752

Kasap SO, Yannacopoulos S (1989) Kinetics of structural relaxations in the glassy semiconductor amorphous selenium. J Mat Res 4:893-905

Krieg NR, Holt JG, Murray RGE (1984) Bergey's manual of systematic bacteriology, vols 1 and 2. Williams and Wilkins, Baltimore

Mayers B, Gates B, Yin Y, Xia Y (2001) Large scale synthesis of monodisperse nanorods of Se/Te alloys through a homogeneous nucleation and solution growth. Adv Mater 13:1380-1384

Miyata T, Kitamura M, Sunagawa I (1978) Theoretical and experimental morphology of monoclinic selenium. Mineral J 9:482-509

Oremland RS, Herbal MJ, Blum JS, Langely S, Beveridge TJ, Ajayan PM, Sutto T, Ellis AV (2004) Structural and spectral features of selenium nanospheres produced by Se-respiring bacteria. Appl Environ Microbiol 70:52-60

Pearce CI, Coker VS, Charnock JM, Pattrick RAD, Mosselmans JFW, Law N, Beveridge TJ, Lloyd JR (2008) Microbial manufacture of calcogenide-based nanoparticles via reduction of selenite using Veillonella atypica: an in situ EXAFS study. Nanotechnology. doi: 10.1088/09574484/19/15/155603

Peled A, Hadziioannou E (1991) Thermal characterization of amorphous selenium films obtained by low-temperature photodeposition. J Mater Sci 26:1769-1774

Ren L, Zhang H, Tan P, Chen Y, Zhang Z, Chang Y, Xu J, Yang F, Yu D (2004) Hexagonal Se nanospheres synthesized via vapor-phase growth. J Phys Chem B 108: $4627-4630$

Takahashi H, Fujikawa T, Konishi T (2006) XAFS study of nanocrystalline selenium embedded in amorphous matrix. eJ Surf Sci Nanotechnol 4:213-218

Tamura K, Dudley J, Nei M, Kumar S (2007) MEGA4: Molecular Evolutionary Genetics Analysis (MEGA) software version 4.0. Mol Biol Evol 24:1596-1599

Thomson JD, Higgins DG, Gibson TJ (1994) CLUSTAL W: improving the sensitivity of progressive multiple sequence alignment through sequence weighting, positions-specific gap penalties and weight matrix choice. Nucleic Acids Res 22:4673-4680

Zhang X, Xie Y, Xu F, Liu X, Zhang S, Tian X (2003) Room temperature rapid growth of monocrystalline selenium nanowires in a polymer buffer system. Solid State Sci 5:525-527

Zhou B, Zhu JJ (2006) A general route for the rapid synthesis of one-dimensional nanostructured single-crystal Te, Se and Se-Te alloys directly from Te or/and Se powders. Nanotechnology 17:1763-1769 